\title{
Fatores associados ao óbito fetal na gestação de alto risco: Assistência de enfermagem no pré-natal
}

\author{
Factors associated with fetal death in high-risk pregnancies: Prenatal nursing care \\ Factores asociados con la muerte fetal en gestación de alto riesgo: Atención de \\ enfermería prenatal
}

\begin{abstract}
Vitória Marion Costa Silva ${ }^{1 *}$, Nicole Helen Freitas Tavares ${ }^{1}$, Milena Bianca da Silva ${ }^{1}$, Izabela Cristina da Silva ${ }^{2}$, Talita Candeias do Rêgo ${ }^{1}$, Douglas Felipe dos Santos Silva ${ }^{1}$, Talita Rebeca dos Santos Silva ${ }^{1}$, Maria Cecília de Jesus Dias ${ }^{1}$, Karoline Virgínia Mendonça Barros ${ }^{1}$, Ana Cristina Farah Abdon da Silva ${ }^{1}$, Luciana Marques Andreto ${ }^{1}$, Eliana Valentim da Silva ${ }^{1}$, Clécia Cristiane da Silva Sales ${ }^{1}$, Silvana Torres de Almeida ${ }^{1}$, Simone Pires Cavalcanti Machado ${ }^{1}$.
\end{abstract}

\section{RESUMO}

Objetivo: Descrever os principais fatores associados ao óbito fetal na gestação de alto risco, contextualizando com a adequação da assistência de enfermagem no pré-natal. Métodos: Estudo de revisão integrativa. Resultados: A análise das publicações selecionadas permitiu a identificação de duas categorias temáticas: Fatores associados ao óbito fetal e a assistência de enfermagem no pré-natal. Quanto à $1^{\text {a }}$ categoria, observou-se nos estudos que a presença de doenças gestacionais aumentou o risco de natimorto incluindo diabetes mellitus, pré-eclâmpsia e oligodrâmnios/polidrâmnios. Todas essas complicações na gravidez estão associadas as restrições de crescimento intrauterino, representando um fator de risco em torno 2,8 vezes maior para ocorrência de natimortos. Quanto a $2^{\underline{a}}$ categoria evidenciou-se uma elevação da cobertura da atenção pré-natal ao longo dos últimos anos em quase todo país, porém as barreiras mais significativas enfrentadas pelos enfermeiros na redução e prevenção da morte fetal foram o acesso materno aos cuidados de saúde e educação durante a gravidez, que pode ser influenciado pela idade materna extrema, baixo nível socioeconômico, falta de apoio, baixa educação em saúde e localização geográfica. Considerações finais: Por ser considerado um tema pouco estudado, devido a sua baixa visibilidade, faz-se necessário novos estudos envolvendo óbito fetal e assistência de enfermagem no pré-natal de alto risco.

Palavras-chave: Morte fetal, Complicações na gravidez, Atenção primária à saúde, Saúde Materno-Infantil.

\section{ABSTRACT}

Objective: To describe the main factors associated with fetal death in high-risk pregnancy, contextualizing with the adequacy of prenatal nursing care. Methods: Integrative review study. Results: The analysis of the selected publications allowed the identification of two thematic categories: Factors associated with fetal death and prenatal nursing care. Regarding the first category, it was observed in the studies that the presence of gestational diseases increased the risk of stillbirth including diabetes mellitus, preeclampsia and oligodramnios / polydraminios. All these complications in pregnancy are associated with intrauterine growth restriction, representing a risk factor around 2.8 times higher for stillbirths. As for the 2 nd category, there was an increase in the coverage of prenatal care over the last years in almost every country, but the most significant barriers faced by nurses in reducing and preventing fetal death were maternal access to health care and education during pregnancy, which may be influenced by maternal age, low socioeconomic status, lack of support, poor health education and geographical location. Final Considerations: Because it is considered a little studied subject, due to its low visibility, further studies involving fetal death and high-risk prenatal nursing care are needed.

Key words: Fetal Death, Complications in pregnancy, Primary Health Care, Maternal and Child Health.

\footnotetext{
${ }^{1}$ Faculdade Pernambucana de Saúde (FPS), Recife - PE. *E-mail: vitoriamarion@hotmail.com

2 Faculdade do Recife (FAREC), Recife - PE.
} 


\section{RESUMEN}

Objetivo: Describir los principales factores asociados con la muerte fetal en el embarazo de alto riesgo, contextualizando con la adecuación de la atención de la enfermería prenatal. Métodos: Estudio de revisión integral. Resultados: El análisis de las publicaciones seleccionadas permitió la identificación de dos categorías temáticas: factores asociados con la muerte fetal y la atención prenatal de enfermería. Con respecto a la primera categoría, se observó en los estudios que la presencia de enfermedades gestacionales aumentaba el riesgo de muerte fetal, incluyendo diabetes mellitus, preeclampsia y oligodramina/ polihidramina. Todas estas complicaciones en el embarazo están asociadas con la restricción del crecimiento intrauterino, lo que representa un factor de riesgo alrededor de 2.8 veces mayor para las muertes fetales. En cuanto a la segunda categoría, hubo un aumento en la cobertura de atención prenatal en los últimos años en casi todos los países, pero las barreras más importantes que enfrentan las enfermeras para reducir y prevenir la muerte fetal fueron el acceso materno a la atención médica y educación durante el embarazo, que puede estar influenciada por la edad materna, el bajo nivel socioeconómico, la falta de apoyo, la mala educación sanitaria y la ubicación geográfica. Consideraciones finales: Debido a su baja visibilidad, considerando un tema poco estudiado, son necesarios más estudios que involucren la muerte fetal y la atención de enfermería prenatal de alto riesgo.

Palabras clave: Muerte Fetal, Complicaciones en el Embarazo, Atención Primaria de salud, Salud maternoinfantil.

\section{INTRODUÇÃO}

A mortalidade fetal apresenta um impacto importante no sentido, por ser considerada um indicador capaz de medir o nível de desenvolvimento de saúde em determinadas regiões do país e por medir a qualidade da assistência prestada a gestante e ao parto (BARROS OS, AQUINO EC, SOUZA MR, 2019). Óbito fetal é definido como a morte de um produto da concepção, expulsão ou da extração completa do corpo da mãe, independentemente da duração gestacional (WORLD HEALTH ORGANIZATION, 2016), evidenciada pela ausência de respiração, de batimentos cardíacos, de pulsações do cordão umbilical ou de movimentos voluntários de contração muscular (ROUQUAYROL MZ, 2018; BRASIL, 2017; WORLD HEALTH ORGANIZATION, 2016).

De acordo com a $10^{\text {a }}$ revisão da Classificação Internacional de Doenças (CID-10) define como óbito fetal precoce os óbitos em fetos com $500 \mathrm{~g}$ ou mais, ou ocorridos a partir de 22 semanas completas de gestação ou mais, ou medindo $25 \mathrm{~cm}$ ou mais de comprimento. (MINISTÉRIO DA SAÚDE BR, 2009; OPAS, 2008). Já os óbitos tardios são aqueles que acontecem a partir das 28 semanas de gestação, em fetos com $1.000 \mathrm{~g}$ ou mais ou com comprimento igual ou maior a $35 \mathrm{~cm}$. Enquanto as perdas gestacionais que ocorrerem em um período abaixo de 22 semanas, são consideradas aborto (LAWN JE, et al., 2016).

Devido as vulnerabilidades socioeconômicas e a inadequada assistência materno-infantil durante o trabalho de parto e parto, estima-se que no mundo ocorrem cerca de 4,9 milhões de mortes perinatais por ano, incluindo 2 milhões de mortes fetais e 2,9 milhões de mortes neonatais prematuras, onde 1 milhão de recém-nascidos morrem no dia que nascem (LAWN JE, et al., 2014; MERALI HS, et al., 2014; MASSON VL, FARQUHAR CM, SADLER LC, 2016; BAQUI AH; MITRA DK; BEGUM N, 2016 e WORLD HEALTH ORGANIZATION, 2016).

Dentre os óbitos fetais, $98 \%$ ocorrem em países de baixa e média renda, principalmente na África subsaariana e no sul da Ásia, incluindo o Nepal (WANG H et al., 2015), além da taxa de mortalidade perinatal apresentar-se cinco vezes maior em países de baixa renda quando comparada com a dos países de alta renda, ficando em torno de 10 mortes por 1.000 nascimentos totais (WORLD HEALTH ORGANIZATION, 2017). Para as nações subdesenvolvidas e em desenvolvimento a natimortalidade tem se tornado uma preocupação constante. Comparando dados de 2000 a 2015, houve progresso, mas a redução ainda continua lenta quando comparada as mortes maternas, neonatais e pós-neonatais, refletindo assim as desigualdades no acesso aos serviços de saúde, além de destacar uma lacuna importante entre ricos e pobres (BRASIL MS, 2018). Estudos têm sinalizado para uma tendência crescente do referido indicador de saúde a nível nacional ao longo do tempo, pois a taxa de mortalidade fetal do país passou de 8,19 em 1996 para 9,50 por 1.000 nascimentos em 2015 (BARROS OS, AQUINO EC, SOUZA MR, 2019). 
Sabe-se que a maior parte dos óbitos perinatais é provocada por causas preveníveis e entre as causas de óbito fetal, destacam-se as de etiologia materna, tanto prévias, tais como obesidade, uso de álcool e drogas, infecções maternas, soropositividade para o HIV-aids (QUIBEL T, et al., 2014), a falta de informação sobre planejamento familiar (KHAN MN, et al., 2017) quanto obstétricas, como doença hipertensiva específica da gestação (DHEG), diabetes mellitus gestacional, as complicações placentárias e amnióticas, incluindo doenças do cordão (como hematoma retroplacentário, estenose de cordão), as malformações fetais, restrição de crescimento fetal e o trauma ao nascimento (QUIBEL T, et al., 2014). Outros fatores associados com óbito fetal que merecem atenção foram: a idade materna de 10 a 14 anos e maior que 35 anos, baixa renda e escolaridade, pré-natal inadequado e natimorto prévio (BARBEIRO FMS, et al., 2015).

Sabe-se que a atenção pré-natal é reconhecida como fator relevante na proteção e na prevenção a eventos adversos sobre a saúde obstétrica, possibilitando a identificação e o manuseio clínico de intervenções oportunas sobre potenciais fatores de risco para complicações à saúde das mães e de seus recém-nascidos (BASSO CG, NEVES ET, SILVEIRA A, 2012). No entanto, as principais falhas que contribuem para as altas taxas de mortalidade fetal e também neonatal precoce estão relacionadas à má qualidade da atenção prénatal, ao diagnóstico tardio de complicações na gravidez, ao manejo obstétrico inadequado e a dificuldade de atendimento a gestante de baixo a alto risco, bem como ao recém-nascido na sala de parto (SANTANA TCP, et al., 2019).

Mesmo diante da magnitude do problema e apesar do lançamento do plano de ação para reduzir as mortes por causas evitáveis e os óbitos fetais até 2035 (ORGANIZAÇÃO MUNDIAL DA SAÚDE, 2014), o referido indicador não foi aceito pela Organização das Nações Unidas (ONU) como um dos Objetivos do Desenvolvimento do Milênio (BLENCOWE H, et al., 2016), o que acarreta em um prejuízo mundialmente relevante em países de maior carência social (WORLD HEALTH ORGANIZATION, 2017).

Diante deste cenário e embora não abrangendo os natimortos alguns programas e políticas de saúde foram criados no Brasil focando na prevenção e redução dos óbitos materno-neonatal, a exemplo a Rede Cegonha e o Programa Nacional de Melhoria do Acesso e da Qualidade da Atenção Básica (PMAQ-AB) (MINISTÉRIO DA SAÚDE BR, 2011; SILVA ACFA, et al., 2019), no qual os óbitos fetais estariam implicitamente incluídos no escopo dessa política, dado a um conjunto de elementos constitutivos que abrange o cuidado na gestação e no parto (BRASIL MS, 2012a).

E por referenciar os cuidados na gestação, em especial o de alto risco (PNAR) o Ministério da Saúde preconiza $O$ atendimento do binômio materno-concepto por uma equipe multidisciplinar, que inclui 0 enfermeiro (LUCIANO MP, SILVA EF, CECCHETTO FH, 2015). Dentre as ações desse profissional de saúde destaca-se a consulta periódica de enfermagem no pré-natal, que permite identificar o mais precocemente possível os problemas reais e potenciais da gestante e, consequentemente, permite elaborar o planejamento das ações de cuidado em decorrência da avaliação de riscos clínicos e obstétricos, além da garantia de acesso a unidade de referência ambulatorial e hospitalar (BRASIL MS, 2015; RODRIGUES ARM, et al., 2016).

O enfermeiro desempenha um papel fundamental, para que ocorra um pré-natal de qualidade já que a equipe de enfermagem deve estar apta a realizar uma assistência humanizada e individualizada, baseada nas ações de acolhimento e de escuta qualificada, levando em consideração as queixas, medos, dúvidas e anseios da gestante (OLIVEIRA DC, et al., 2017). Para tanto, o objetivo do estudo foi descrever os principais fatores associados ao óbito fetal na gestação de alto risco, contextualizada com a adequação da assistência de enfermagem no pré-natal.

\section{METODOLOGIA}

Trata-se de uma revisão integrativa, com levantamento bibliográfico realizado no período de março à setembro de 2019, através de consulta direta pela internet, no endereço eletrônico da plataforma da Biblioteca Virtual de Saúde (BVS), onde buscou-se por publicações cientificas indexadas nas seguintes bases de dados: Literatura Latino-Americana e do Caribe em Ciências da Saúde (LILACS); Scientific Electronic Library Online (SciELO); Base de Dados Nacionais da Enfermagem (BDENF); Cumulative Index to Nursing and Allied Health 
Literature (CINAHL); ), Elsevier's Abstract and Itation Database (Scopus); Science Citation Index (Web of Science); US National Library of Medicine National Institutes of Health (Medline/PubMed) e Índice Bibliográfico Español de Ciencias de la Salud (IBECS) (Figura 1).

Através do acesso on-line, foram utilizados os Descritores em Ciências da Saúde (DeCS): Morte fetal, Morte perinatal, Enfermagem perinatal, Complicações da gravidez, Atenção primária de saúde, Saúde materno-infantil, além dos descritores cadastrados no Medical Subject Headings (MesH), artigos na íntegra publicados em português, inglês e espanhol que abordassem a temática em estudo independentemente do método de pesquisa utilizado e no mínimo de cinco anos de publicação.

Para guiar a revisão formulou-se a seguinte questão norteadora: quais são os instrumentos existentes na literatura nacional e internacional que podem ser utilizados para avaliar a saúde materno-infantil, através da adequação da assistência de enfermagem no pré-natal de alto risco, visando a redução dos óbitos fetais? $\mathrm{Na}$ elaboração da pergunta e na busca de evidencias científicas utilizou-se a estratégia PICO (Quadro 1), uma sigla que possui o seguinte significado: "P" de paciente ou população; "l" de intervenção ou indicador; "C" de comparação ou controle; e "O" de outcome, que na língua inglesa significa desfecho clinico, resultado ou, ainda, a resposta que se espera encontrar nos estudos selecionados. Entretanto, nesse estudo não se fez ressalva ao elemento $(C)$, posto que a referida pesquisa não visa realizar comparação entre as intervenções.

Quadro 1 - Estratégia de busca nas bases de dados.

\begin{tabular}{|c|c|c|c|}
\hline PICO & DeCS & MESH & TíTULOS CINAHL \\
\hline P & $\begin{array}{c}\text { Morte perinatal OR Morte } \\
\text { fetal OR Natimorto }\end{array}$ & $\begin{array}{c}\text { Perinatal death OR Fetal } \\
\text { death OR Stillbirth }\end{array}$ & $\begin{array}{c}\text { Perinatal death OR Fetal } \\
\text { death OR Stillbirth }\end{array}$ \\
\hline I & AND & AND & AND \\
\hline C & $\begin{array}{c}\text { Assistência perinatal OR } \\
\text { Cuidados de enfermagem } \\
\text { OR Assistência antenatal }\end{array}$ & $\begin{array}{c}\text { Perinatal Care OR Nursing } \\
\text { Care OR Obstetric Nursing }\end{array}$ & $\begin{array}{c}\text { Perinatal Care OR Nursing } \\
\text { Care OR Obstetric Nursing }\end{array}$ \\
\hline O & & & \\
\hline & Saúde Materno-Infantil & Maternal and Child Health & Maternal and Child Health \\
\hline
\end{tabular}

Fonte: Silva VMC, et al.,2019.

Foi necessário o uso do software EndNote ${ }^{\circledR}$ para importar os artigos das bases de dados, organizando-os em dois grupos chamados: "selecionados para leitura na íntegra" e "excluídos após leitura de título, resumo e artigo complete". Para a pesquisa foram utilizados descritores (DECS/MESH) e termos livres (MESH/TERMS), cruzados através do operador booleano "OR" dispostos na (Quadro 2).

Quadro 2 - Estratégias de busca dos artigos para consulta em bases de dados eletrônicas.

\begin{tabular}{|l|l|l|}
\hline Cruzamento em inglês & Cruzamento em espanhol & Cruzamento em português \\
\hline Fetal Death (DECS/MESH) OR & Muerte Fetal (DECS/MESH) OR & Morte fetal (DECS/MESH) OR \\
Perinatal Death (MESH TERMS), & Muerte Perinatal (MESH & Morte perinatal (MESH TERMS), \\
OR Pregnancy Complication & TERMS), OR Complicaciones del & OR Complicações da gravidez \\
(MESH TERMS). & Embarazo (MESH TERMS). & (MESH TERMS). \\
\hline Neonatal Nursing (DECS/MESH) & Enfermería neonatal & $\begin{array}{l}\text { Enfermagem perinatal } \\
\text { OR Prenatal Care (MESH }\end{array}$ \\
TERMS) OR Fetal Death (MESH & Prenatal (MESH TERMS) OR & (DECS/MESH) OR Assistência \\
pré-natal (MESH TERMS) OR \\
TERMS). & Muerte Fetal (MESH TERMS). & Morte fetal (MESH TERMS). \\
\hline Perinatal Death (DECS/MESH) & Muerte Perinatal (DECS/MESH) & Morte perinatal (DECS/MESH) \\
OR Maternal and Child Health & OR Salud materno-infantil & OR Saúde Materno-infantil \\
(MESH TERMS), OR Primary & (MESH TERMS), OR Atención & (MESH TERMS), OR Atenção \\
Health Care (MESH TERMS). & Primaria a la salud (MESH & Primária de Saúde (MESH \\
& TERMS). & TERMS). \\
\hline
\end{tabular}

Fonte: Silva VMC, et al., 2019. 
Para realização desse trabalho foram incluídos artigos originais, disponíveis na íntegra e de revisão sistemática sobre a temática em questão: principais fatores associados ao óbito fetal na gestação contextualizando com a assistência de enfermagem perinatal, sem restrição de idiomas ou país de publicação. Os artigos nos diversos idiomas foram traduzidos por um professor de língua estrangeira da própria instituição de ensino quando necessário. Foi realizado um recorte temporal de 2015 a 2019, disponíveis nas bases de dados eletrônicas.

Como critério de exclusão decidiu-se por não utilizar artigo que não estava ajustado ao objeto de estudo, que fugiram do tema proposto pelos autores, os que se encontravam duplicados nas bases de dados eletrônicas, textos que encontram-se incompletos, indisponível na íntegra, as revisões de literatura, guias, manuais técnicos e cartas ao leitor.

Para a escolha dos artigos, foi necessário que os autores respeitassem os critérios de inclusão e exclusão. Fez-se necessário que os pesquisadores realizassem a releitura dos títulos, resumos e descritores e se houvesse alguma incerteza sobre sua inclusão, se realizou a leitura completa dos artigos e mesmo diante de todas as etapas percorridas porventura permanecesse a dúvida, se optou pela escolha de dois revisores que continham pleno conhecimento dos critérios de inclusão/exclusão que de forma independente e em duplo cego realizou o cruzamento dos descritores por meio do operador booleano "OR" em prol de uma segunda e última análise e se mesmo diante da conclusão de todas essas etapas, se ainda permanecesse a dúvida, se utilizou a técnica de duplo consenso.

\section{RESULTADOS}

Inicialmente, foram identificados 675 artigos e destes 130 foram selecionados para o estudo, provenientes de periódicos internacionais (40\%) e nacionais (60\%), disponíveis nos bancos de dados eletrônicos. Também foram excluídos os artigos duplicados (59), ou seja, que se encontravam repetidos nas bases de dados eletrônicas, como LILACS, CINAHL, BDENF, SCOPUS, MEDLINE via PUBMED, WEB OF SCIENC, SCIELO e IBECS, ficando uma amostra parcial de 26 artigos, onde foram novamente filtrados e eliminados por critérios de exclusão (19), obtendo-se, assim, uma amostra final de sete estudos nacionais $(n=7)$, representados por dois internacionais e cinco nacionais referentes ao tema do projeto de acordo com a Tabela 2.

Tabela 2 - Número de artigo obtidos nas bases de dados eletrônicas no período de março a setembro de 2019.

\begin{tabular}{ccccccc}
\hline $\begin{array}{c}\text { Bases de } \\
\text { Dados }\end{array}$ & $\begin{array}{c}\text { Artigos } \\
\text { encontrados }\end{array}$ & $\begin{array}{c}\text { Artigos } \\
\text { selecionados }\end{array}$ & $\begin{array}{c}\text { Artigos } \\
\text { duplicados }\end{array}$ & $\begin{array}{c}\text { Artigos } \\
\text { excluídos }\end{array}$ & $\begin{array}{c}\text { Parcial artigos } \\
\text { analisados }\end{array}$ & $\begin{array}{c}\text { Amostra } \\
\text { final }\end{array}$ \\
\hline LILACS & 237 & 71 & 31 & 21 & 19 & 3 \\
CINAHL & 33 & 11 & 7 & 4 & 0 & 0 \\
SCOPUS & 26 & 8 & 4 & 4 & 0 & 0 \\
MEDLINE & 98 & 17 & 7 & 7 & 3 & 2 \\
Web of & 53 & 9 & 4 & 3 & 2 & 0 \\
Science & & 4 & 2 & 1 & 1 & 1 \\
SCIELO & 187 & 5 & 2 & 2 & 1 & 1 \\
BDEHF & 19 & 5 & 2 & 3 & 0 & 0 \\
IBECS & 22 & 130 & 59 & 45 & 26 & 7 \\
\hline TOTAL & 675 & & & & &
\end{tabular}

Fonte: Silva VMC, et al.,2019. 
Para aumentar a quantidade de revisões sistemáticas e a confiabilidade da pesquisa foi usado um documento Preferred Reporting Items for Systematic Reviews and Meta-Analysis (PRISMA), disposto na Figura 1.

Figura 1 - Fluxograma com estratégias de seleção de artigos, após busca em bases de dados eletrônicos, 2015 - 2019, segundo modelo PRISMA.

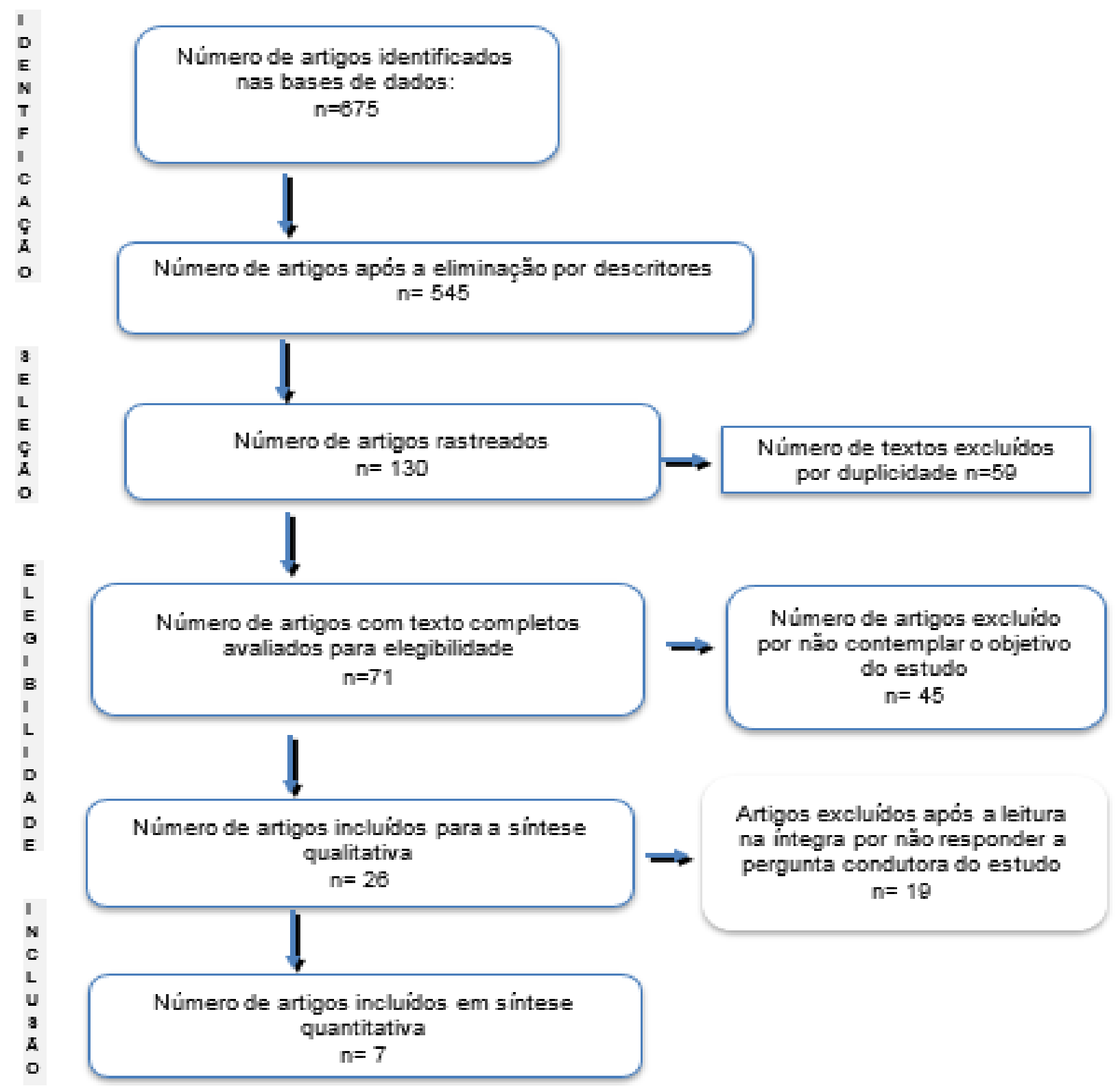

Fonte: Silva VMC, et al.,2019.

As publicações foram analisadas, os dados interpretados de forma organizada e sintetizada por meio da construção de uma tabela com descrição dos seguintes aspectos: Autores/ano, desenho/objetivo metodológico, nível de evidência, intervenção, resultados e conclusão, dispostos na Tabela 2.

De acordo com a classificação, as pesquisas enquadram-se como artigos originais $(n=7)$, sendo todos de abordagem quantitativa, porém apresentando diferentes desenhos metodológicos. 


\section{Revista Eletrônica Acervo Saúde / Electronic Journal Collection Health ISSN 2178-2091}

Tabela 2 - Análise de artigos sobre mortalidade neonatal precoce e fatores de risco materno-infantil, 2015 - 2019.

\begin{tabular}{|c|c|c|c|c|c|c|}
\hline Autores/ano & Metodologia & Objetivo & $\begin{array}{l}\text { Nível de } \\
\text { evidência }\end{array}$ & Intervenção estudada & Resultados & Conclusões \\
\hline $\begin{array}{l}\text { Barbeiro FMS, } \\
\text { et al., (2015). }\end{array}$ & $\begin{array}{l}\text { Revisão } \\
\text { sistemática de } \\
\text { estudos } \\
\text { brasileiros sobre } \\
\text { óbitor fetal } \\
\text { publicados entre } \\
2003 \text { e } 2013\end{array}$ & $\begin{array}{l}\text { Analisar frequência e } \\
\text { fatores associados ao } \\
\text { óbito fetal na literatura } \\
\text { científica brasileira. }\end{array}$ & $\underset{\star \star \star \star \star * *}{\text { Nível }} 6$ & $\begin{array}{l}\text { Qualidade da informação, } \\
\text { estudo descritivo e } \\
\text { fatores associados. }\end{array}$ & $\begin{array}{l}\text { Os principais fatores } \\
\text { associados ao óbito fetal } \\
\text { foram pré-natal } \\
\text { inadequado ou ausente, } \\
\text { baixa escolaridade, } \\
\text { morbidades maternas e } \\
\text { história reprodutiva } \\
\text { desfavorável }\end{array}$ & $\begin{array}{l}\text { O pré-natal deve priorizar } \\
\text { mulheres mais vulneráveis, } \\
\text { socialmente ou em relação à } \\
\text { história reprodutiva e } \\
\text { morbidades, para diminuir a } \\
\text { taxa de mortalidade fetal no } \\
\text { Brasil. }\end{array}$ \\
\hline $\begin{array}{l}\text { Nunes JT, et } \\
\text { al., (2016). }\end{array}$ & $\begin{array}{lr}\text { Revisão } & \text { de } \\
\text { sistemática } & \\
\text { conduzida } & \text { a } \\
\text { partir } & \text { de } \\
\text { recomendações } \\
\text { propostas pelo } \\
\text { protocolo } \\
\text { PRISMA }\end{array}$ & $\begin{array}{l}\text { Investigar a qualidade } \\
\text { da atenção pré-natal no } \\
\text { Brasil de } 2005 \text { a } 2015 \\
\text { em relação ao acesso e } \\
\text { à adequação da } \\
\text { assistência prestada. }\end{array}$ & $\underset{* * * * * a}{\text { Nível }} 6$ & $\begin{array}{c}\text { Qualidade da } \\
\text { investigação foi realizada } \\
\text { através do Cheklist for } \\
\text { Measuring Quality. }\end{array}$ & $\begin{array}{l}\text { Verificaram-se } \\
\text { variações na adequação } \\
\text { da assistência pré-natal, } \\
\text { especialmente, quando } \\
\text { comparadas à aplicação } \\
\text { de diferentes } \\
\text { indicadores } \\
\text { qualidade }\end{array}$ & $\begin{array}{l}\text { A atenção pré-natal do Brasil } \\
\text { da última década não pode } \\
\text { ser considerada adequada, } \\
\text { visando a } \begin{array}{r}\text { melhora } \\
\text { significativa em vários } \\
\text { aspectos da assistência, } \\
\text { principalmente de forma } \\
\text { qualitativa. }\end{array}\end{array}$ \\
\hline $\begin{array}{l}\text { Demitto MO, et } \\
\text { al (2017). }\end{array}$ & $\begin{array}{l}\text { Estudo } \\
\text { epidemiológico } \\
\text { do tipo } \\
\text { transversal. }\end{array}$ & $\begin{array}{lr}\text { Identificar os } & \text { fatores } \\
\text { associados } & \text { à } \\
\text { mortalidade neonatal } \\
\text { intra-hospitalar com } \\
\text { base nas características } \\
\text { individuais de gestantes } \\
\text { de risco. }\end{array}$ & Nível 4 & $\begin{array}{l}\text { Modelo descritivo de } \\
\text { peregrinação das mães } \\
\text { até o parto. }\end{array}$ & $\begin{array}{l}\text { Trabalho de parto } \\
\text { prematuro, } \\
\text { malformação fetal e } \\
\text { gestação múltipla foram } \\
\text { as intercorrências } \\
\text { associadas ao óbito } \\
\text { materno-fetal } \\
\text { neonatal. }\end{array}$ & $\begin{array}{l}\text { A identificação de fatores de } \\
\text { risco pode auxiliar no } \\
\text { planejamento de ações para } \\
\text { consolidação da rede } \\
\text { perinatal e assim contribuir } \\
\text { para as melhorias dos } \\
\text { indicadores. }\end{array}$ \\
\hline $\begin{array}{lr}\text { Sampaio } & \text { AFS, } \\
\text { Rocha } & \text { MJF, } \\
\text { Leal } & \text { EAS } \\
(2018) . & \end{array}$ & $\begin{array}{l}\text { Estudo do tipo } \\
\text { corte } \\
\text { transversal }\end{array}$ & $\begin{array}{l}\text { Descrever o perfil } \\
\text { clínico e epidemiológico } \\
\text { das gestantes } \\
\text { atendidas no serviço de } \\
\text { pré-natal de alto risco } \\
\text { da Maternidade Pública } \\
\text { de Rio Branco, Acre }\end{array}$ & Nível 4 & $\begin{array}{c}\text { Entrevista e aplicação de } \\
\text { questionário. }\end{array}$ & 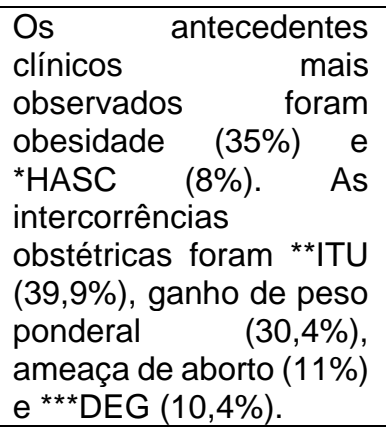 & $\begin{array}{l}\text { O conhecimento acerca do } \\
\text { perfil clínico epidemiológico } \\
\text { das gestantes de alto risco } \\
\text { permite auxiliar na criação } \\
\text { de instrumentos estratégicos } \\
\text { dos serviços de saúde e } \\
\text { consequentemente na } \\
\text { redução da mortalidade } \\
\text { materna e fetal. }\end{array}$ \\
\hline
\end{tabular}




\section{Revista Eletrônica Acervo Saúde / Electronic Journal Collection Health ISSN 2178-2091}

\begin{tabular}{|c|c|c|c|c|c|c|}
\hline $\begin{array}{l}\text { Santana TCP, } \\
\text { et al., (2019). }\end{array}$ & $\begin{array}{l}\text { Estudo do tipo } \\
\text { revisão } \\
\text { integrativa, de } \\
\text { abordagem } \\
\text { quantitativa }\end{array}$ & $\begin{array}{l}\text { Avaliar o impacto do } \\
\text { indicador } \\
\text { morbimortalidade } \\
\text { materno-neonatal, } \\
\text { através das dificuldades } \\
\text { vivenciadas por } \\
\text { enfermeiros no pré- } \\
\text { natal. }\end{array}$ & $\underset{* \star * \star * *}{\text { Nível } 4}$ & $\begin{array}{l}\text { Levantamento } \\
\text { bibliográfico. }\end{array}$ & $\begin{array}{l}\text { Algumas dificuldades } \\
\text { alancadas ao pré-natal } \\
\text { de risco habitual, tais } \\
\text { como, ausência de } \\
\text { referência } \\
\text { contrarreferência, } \\
\text { carência de recursos } \\
\text { materiais-tecnológicos, } \\
\text { espaço físico da } \\
* * * \text { UBS. }\end{array}$ & $\begin{array}{l}\text { Há necessidade do } \\
\text { desenvolvimento de outras } \\
\text { pesquisas que aprofundem } \\
\text { sobre o tema, visando } \\
\text { minimizar as taxas de } \\
\text { morbimortalidade em seu } \\
\text { componente } \\
\text { neonatal. }\end{array}$ \\
\hline $\begin{array}{l}\text { Martins MCF, } \\
\text { et al (2019). }\end{array}$ & $\begin{array}{l}\text { Estudo do tipo } \\
\text { caso-controle e } \\
\text { de abordagem } \\
\text { prospectiva. }\end{array}$ & $\begin{array}{l}\text { ldentificar a associação } \\
\text { entre os atrasos nos } \\
\text { cuidados prestados as } \\
\text { gestantes e os } \\
\text { desfechos de morte } \\
\text { fetal. }\end{array}$ & Nível 4 & $\begin{array}{l}\text { Consultas de pré-natal, } \\
\text { contextualizando com três } \\
\text { categorias de atrasos na } \\
\text { assistência. }\end{array}$ & $\begin{array}{l}\text { O grupo com morte fetal } \\
\text { teve um número mínimo } \\
\text { de consultas de pré- } \\
\text { natal, menor orientação } \\
\text { dos serviços de saúde } \\
\text { para o parto, consulta } \\
\text { inadequada, ausência } \\
\text { do pré-natal, dentre } \\
\text { outros. }\end{array}$ & $\begin{array}{l}\text { A prevenção de natimortos } \\
\text { não pode ser um tema } \\
\text { individualizado, estratégias } \\
\text { para redução dessas mortes } \\
\text { requer ações coordenadas e } \\
\text { contínuas nos diversos } \\
\text { níveis de atenção. }\end{array}$ \\
\hline $\begin{array}{l}\text { Silva ACFA, al., } \\
(2019)\end{array}$ & $\begin{array}{l}\text { Estudo do tipo } \\
\text { revisão } \\
\text { integrativa. }\end{array}$ & $\begin{array}{l}\text { Identificar os principais } \\
\text { fatores de risco que } \\
\text { contribuem para a } \\
\text { mortalidade neonatal } \\
\text { precoce a partir do perfil } \\
\text { materno. }\end{array}$ & $\underset{* \star \star \star * \star}{\text { Nível } 6}$ & $\begin{array}{l}\text { Levantamento } \\
\text { bibliográfico. }\end{array}$ & $\begin{array}{l}\text { Fatores associados ao } \\
\text { óbito neonatal, tais como, } \\
\text { o índice de vitalidade } \\
\text { neonatal menor que sete, } \\
\text { peso ao nascer, inferior a } \\
1.500 \text { e a má formação } \\
\text { congênita, além da Idade } \\
\text { gestacional menor que } 30 \\
\text { semanas. }\end{array}$ & $\begin{array}{l}\text { Estudos evidenciam a } \\
\text { necessidade de rever o } \\
\text { acesso aos serviços de saúde } \\
\text { em todo território brasileiro, } \\
\text { assim como a qualidade da } \\
\text { assistência no pré-natal, e, as } \\
\text { condições de assistência ao } \\
\text { parto. }\end{array}$ \\
\hline
\end{tabular}

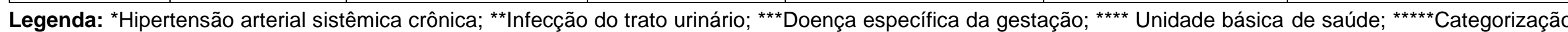

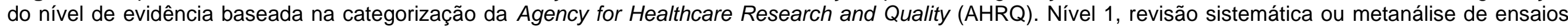

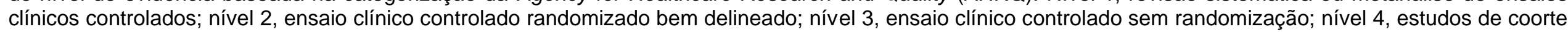

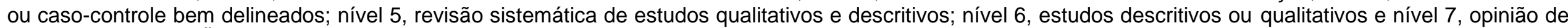
especialistas (GALVÃO CM, 2006).

Fonte: Silva VMC, et al.,2019. 


\section{DISCUSSÃO}

O Brasil apresenta níveis alarmantes de mortalidade materno-fetal relacionados a problemas perinatais, nos quais algumas sub-regiões apesar de taxas apresentarem em declínio nos últimos dez anos, ainda estamos longe da condição aceitável (CANUTO IMB, 2019). O que foi observado nas últimas décadas em diversos países do mundo é a baixa diminuição ou a precária estabilidade nos coeficientes de mortalidade fetal (FROEN JF, et al., 2016; PARIS GF; DEMONTIGNY F; PELLOSO SM, 2016). Regionalmente, as taxas de natimortos foram mais altas entre os países da África subsaariana central, que excedeu 23 óbitos por 1.000 nascimentos em 2016. As taxas foram altamente variáveis em todo o sul e sudeste da Ásia, de 3,5 óbitos por 1.000 nascimentos na Malásia a 25,9 óbitos por 1.000 nascimentos no Paquistão (GLOBAL BURDEN OF DISEASES, 2016).

Entre 1970 e 2016, os resultados de natimortos por idade específica, por mil nascidos vivos na Europa Central, Europa Oriental e na Ásia foram de 4,5; 6,3 e 6,8, respectivamente, havendo um aumento considerado na África do Norte com 17,4 e Oriente Médio com 18,1. Enquanto o Brasil, no período de 2000 a 2016, a taxa de óbitos fetais foram de 5,3, porém por regiões a curva estacionária apresentou uma tendência crescente, quando associada a escolaridade da mãe, duração da gestação e tipo de parto (GLOBAL BURDEN OF DISEASES, 2016). Segundo dados do departamento de informática do Sistema Único de Saúde (DATASUS), (2017), o Brasil apresentou um total de 30.620 casos de óbitos fetais, sendo distribuídos da seguinte maneira por região: Norte (3.483); Nordeste (10.199); Sudeste (11.419); Sul (3.186) e Centro-Oeste (2.333). Em Pernambuco (1.513) e em Recife (216).

A taxa de natimortalidade não é uma questão restrita aos países em desenvolvimento, posto que entre os países desenvolvidos, há uma variação nos indicadores de mortes fetais que poderiam ser evitadas, tais como fatores relacionados ao nascimento, como, o baixo peso ao nascer (DEMITTO MO, 2017), a prematuridade, o índice de apgar $\leq a$ 7, asfixia intraparto, a hipóxia, a termorregulação ineficaz (SILVEIRA RC, 2013; LANSKY SC, 2014), bem como, fatores relacionados a gestante, como, a baixa idade gestacional, as infecções perinatais diversas (LOURENÇO N, et al., 2017), sífilis congênita (VIELLAS EF, et al.,2014), diabetes gestacional, eclampsia (LASSI ZS, 2015) e fatores estruturais, dentre eles, a falta de preparo das gestantes para o parto e aleitamento, além das dificuldades de acesso aos exames e realização de consultas (BRASIL MS, 2017; LIVRAMENTO DVP et al., 2019).

Na maioria das situações, as consultas de enfermagem tanto de baixo como de alto risco contemplaram a prática do exame físico obstétrico completo, aferição de peso e pressão arterial, medição da altura uterina, ausculta de batimentos cardíacos fetais quando em idade gestacional adequada ao exame, acompanhamento dos resultados de todos os exames solicitados a cada trimestre gestacional, conforme preconizado pelo Ministério da Saúde (2017). Em contrapartida para Mayor MSS e colaboradores (2018), a assistência prénatal não deve se reduzir apenas à realização de consultas e solicitação de exames, pois precisa considerar também 0 acolhimento e o reconhecimento das necessidades singulares das gestantes, visando o estabelecimento de vínculos entre o profissional-usuária.

Segundo a Política Nacional de Humanização MS (2006) "acolhimento" implica prestar um atendimento com resolutividade e corresponsabilização, orientando, conforme o caso, o usuário e a família, garantindo a articulação com os outros serviços de saúde para a continuidade da assistência quando necessário", incluindo a maternidade programada para a realização do parto.

Sabe-se que a qualidade da assistência pré-natal "impacta diretamente nos indicadores de saúde de morbimortalidade materna e perinatal, entretanto também contribui para a redução dessa taxa (MAYOR MSS, et al., 2018), porém ainda existem gestantes classificadas com assistência ante parto inadequada, cabendo ao enfermeiro realizar consultas de pré-natal de baixo risco ou de risco habitual (CARDOSO MD, et al., 2016), além de acompanhar o de alto risco, assegurado conforme a lei do exercício profissional de ำ 7.498/86.

Alguns estudos mostram que o indicador de saúde ainda mantém um caráter persistente, sendo um dos motivos, o quadro de iniquidade social e as persistentes desigualdades intraurbanas (UNICEF, 2017; CANUTO IMB, et al., 2019), dados esses também que foram enfatizados em outro estudo ao retratar que o 
país pouco apresentou mudanças nos principais determinantes e condicionantes sociais e na organização dos serviços de saúde, quanto ao acesso a maioria das intervenções em saúde materno-infantil, devido a maior complexidade dos fatores biológicos, socioeconômicos e assistenciais (LEAL MC, et al., 2018). Segundo Migoto MT (2018), considera que tanto o acesso, como a qualidade dos serviços de saúde são diretamente impactados pelos fatores socioeconômicos e culturais de uma população e isso reflete não somente em prejuízo à saúde materna-fetal e neonatal, como também, pode resultar em maiores taxas de complicações gestacionais, ou até mesmo natimortalidade.

Quanto aos óbitos perinatais, Lassi ZS e Bhutta ZA (2015) relataram que a faixa etária materna entre 20 e 34 anos e a baixa escolaridade das mães são considerados elementos relevantes, assim como os níveis socioeconômicos mais baixos, corroborando com os dados encontrados em outro estudo (VOGEL JP et al., 2014). Já para um estudo específico sobre a idade materna avançada, ou seja, acima de 35 anos também foi associada a um risco aumentado de morte fetal por asfixia intraparto a termo e a resultados adversos maternos graves (PASUPATHY D, et al., 2011; LEOPAIBOON M, et al., 2014).

Entretanto, para outros autores a presença de intercorrências clínicas na gestação pode predizer resultados perinatais adversos, com possível desfecho em morte fetal ou neonatal (Kumar GA et al., 2014), corroborando com outros artigos que incluem mau passado obstétrico, curto intervalo intraparto, gravidez múltipla, gravidez ectópica, menor idade gestacional, história de natimorto, hipertensão, diabetes, HIV e ausência de cuidados pré-natais, gravidez pós-termo (VOGEL JP, et al., 2014; DEMITTO MO, GRAVENA AAF e ANTUNES MB, 2017).

Nesse contexto, vários esforços e estratégias vêm sendo implementadas, a fim de assegurar uma adequada atenção pré-natal à essa população, visto que existe uma relação entre uma assistência qualificada e os índices de morte materna e fetal (MARTINELLI KG, et al., 2014). Sabemos que toda gestação requer uma atenção pré-natal qualificada, a mulher deve ser provida de uma escuta ativa, além de ações de prevenção e promoção da saúde para a identificação precoce dos fatores de risco, realizada pela equipe de saúde, em evidência a enfermagem (ALVES DP, et al, 2015; GAIOSO SEM, et al., 2014). Em contrapartida pouco mais da metade realizaram de 3 a 5 consultas de pré-natal, estando em desacordo com o preconizado pela Rede Cegonha (BRASIL MS, 2011), posto que seis consultas é a quantidade mínima ideal para a garantia de desfechos clínicos maternos e fetais favoráveis (VIELLAS EF, et al., 2014; SAMPAIO AFS, ROCHA MJF, LEAL EAS, 2018).

A maioria dos estudos publicados, aponta para dificuldades na captação precoce das gestantes que deve ocorrer em até 120 dias da gestação (DARMONT MQR, et al, 2010; BRASIL MS, 2014; MARTINS PQM, et al, 2015), além da não adesão das mesmas ao pré-natal, que pode ser reflexo tanto da falta de informação ou insatisfação, quanto da falta de acolhimento de alguns enfermeiros, ou até mesmo por fatores socioeconômicos maternos, tais como, a baixa renda familiar, a escolaridade, localização geográfica da residência ao serviço de saúde, a qualidade dos cuidados em saúde e de suporte social (LEAL NJ et al, 2018).

Por outro lado, Neumann (2003) retrata que a qualidade da assistência realizada durante o pré-natal pode reduzir de $10 \%$ a $20 \%$ a quantidade de óbitos nesse período, corroborando com outras pesquisas, em que os dados apontam que a adequada atenção pré-natal, tem se apresentado como um dos principais fatores de proteção, não somente contra a mortalidade nessa população em estudo, mas também contra o baixo peso ao nascer, a prematuridade e o retardo do crescimento intrauterino (DARMSTADT GL, 2005; BARROS FC, 2008).

O controle realizado no pré-natal é imprescindível para detecção precoce de intercorrências e realização de ações de prevenção de doenças. É o momento de reconhecer doenças que podem acometer o feto e a mãe durante a gestação, as quais podem levar à prematuridade extrema e consequentemente morte neonatal precoce (RODRIGUES M, 2011).

Para tanto, faz-se necessário, identificar também outros fatores relacionados não somente ao pré-natal, mas ao parto, tais como, idade gestacional, multiparidade, condições e tipo de parto, intervalo intraparto, hemorragia perinatal; mortalidade perinatais, dentre outros (SILVEIRA RC, 2013; LANSKY SC, 2014). 
O estudo mostrou a importância da identificação de fatores de risco, capaz de auxiliar no planejamento de ações para consolidação da rede assistencial perinatal, com reestruturação e qualificação dos cuidados prénatal da gestante de alto risco em busca de resultados expressivos, como a redução da mortalidade fetal.

\section{CONSIDERAÇÕES FINAIS}

Conclui-se que, apesar de a mortalidade fetal apresentar um impacto importante para a saúde pública, por se tratar de um indicador capaz de medir o nível de desenvolvimento de saúde em determinadas regiões do país, e por medir a qualidade da assistência prestada a gestante e ao parto, o risco de morte fetal pode ser evitado se for identificado precocemente os fatores de risco relacionados no pré-natal, pois facilita na hora da tomada de decisão do profissional de saúde para o planejamento da assistência à gestante e feto, com cuidados específicos à gestação de alto risco, para obter resultados satisfatórios, tal como a reduzir a alta taxa de mortalidade fetal.

\section{REFERÊNCIAS}

1. LAWN JE, et al. Ending Preventable Stillbirths Series study group; Stillbirth Epidemiology investigator group. Stillbirths: rates, risk factors, and acceleration towards 2030. Lancet. 2016;387(10018):587-603

2. LAWN JE, et al. Every Newborn: progress, priorities, and potential beyond survival. Lancet. 2014; 384:189-205

3. BAQUI AH, et al. Neonatal mortality within 24 hours of birth in six low- and lower-middle-income countries. Bull World Health Organ. 2016; 94:752. 78b

4. LAWN JE, et al. Stillbirths: rates, risk factors, and acceleration towards 2030. Lancet. 2016; 387:587-603

5. WANG $\mathrm{H}$, et al. Global, regional, national, and selected subnational levels of stillbirths, neonatal, infant, and under-5 mortality, 1980-2015: a systematic analysis for the Global Burden of Disease Study 2015. Lancet. 2016; 388:1725.

6. World Health Organization. Estimates Developed by the UN Inter-Agency-Group for Child Mortality Estimation. Report, 2017. Reprod. 2014;43(10):883- 907.

7. KHAN MN, et al. Maternal undernutrition and excessive body weight and risk of birth and health outcomes. Arch Public Health. 2017; 75:12.

8. BARROS PS, et al. Mortalidade fetal e os desafios para a atenção à saúde da mulher no Brasil. Rev. Saúde Pública vol.53. São Paulo, 2019.

9. BARBEIRO FMS, et al. Óbitos fetais no Brasil: revisão sistemática. Rev Saúde Pública 2015; 49:22.

10. SILVA ACF, et al. Índice de morte neonatal precoce: Uma análise do perfil materno. Revista Eletrônica Acervo Saúde, 2019; (26), e690.

11. BRASIL. Ministério da Saúde. Secretaria de Vigilância em Saúde. Departamento de Análise da Situação em Saúde. Saúde Brasil 2011: uma análise da situação de saúde e a vigilância da saúde da Mulher. Brasília: Ministério da Saúde, 2012a. p. 163-182.

12. BRASIL. Ministério da Saúde. Secretaria de Ciência, Tecnologia e Insumos Estratégicos. Departamento de Ciência e Tecnologia. Síntese de Evidências para Políticas de Saúde: reduzindo a mortalidade perinatal / Ministério da Saúde, Secretaria de Ciência, Tecnologia e Insumos Estratégicos, Departamento de Ciência e Tecnologia. - 3. ed. - Brasília: Ministério da Saúde, 2016. $44 \mathrm{p}$

13. WORLD HEALTH ORGANIZATIVE. The WHO aplicativo of ICD-10 to debates durinhos the perinatal período: ICDPM. Genova: WHO; 2016

14. MERALI HS, et al. Audit-identified avoidable factors in maternal and perinatal deaths in low resource settings: a systematic review. BMC Pregnancy Childbirth [Internet]. 2014 [cited 2017 Oct 20]; 14(280):1-12.

15. MASSON VL, et al. Validation of local review for the identification of contributory factors and potentially avoidable perinatal deaths. Aust N Z J Obstet Gynaecol [Internet]. 2016 [cited 2017 Oct 18]; 56(3):282-8.

16. BLENCOWE H, et al. Measuring maternal, fetal and neonatal mortality: challenges and solutions. Best Pract Res Clin Obstet Gynaecol [Internet]. 2016 [cited 2017 Aug 20]; 36:14-29.

17. SANTANA TCP, et al. Dificuldades dos enfermeiros no atendimento ao pré-natal de risco habitual e seu impacto no indicador de morbimortalidade materno-neonatal. Revista Eletrônica Acervo Saúde, (20), e711.

18. BASSO CG, et al. Associação entre realização de pré-natal e morbidade neonatal. Texto Contexto Enferma. 2012;21(2):269-76

19. DEMITO MO, et al. Gestação de alto risco e fatores associados ao óbito neonatal. Rev. esc. Enfermo. USP vol.51 São Paulo 2017 Pub. Par 03, 2017. 
20. BALSAMAI MA, et al. Intrapartum fetal and Maternal Complications in Low-Risk Pregnancy: Experience of a Tertiary Hospital in Low-Income Countries. Open Access Maced J Med Sci. 2019 Jun 30; 7(12):1979-1983

21. FLENADY V, et al. Stillbirths: recall to action in high-income countries. Lancet. 2016;387(10019):691-702.

22. FROEN JF, et al.; Lancet Ending Preventable Stillbirths Series study group. Stillbirths: progress and unfinished business. Lancet. 2016;387(10018):574-86.

23. PARIS GF, et al. Adaptação transcultural e evidências de validação da Perinatal Grief Scale. Texto Contexto Enferm. 2016;25. No prelo.

24. CANUTO IMB, et al. Diferenciais intraurbanos da mortalidade perinatal: modelagem para identificação de áreas prioritárias. Recife. Escola Anna Nery 2019; 23(1): 1-8.

25. LEAL NJ, et al. Assistência ao pré-natal: depoimento das enfermeiras. Rev Fund Care Online. 2018 jan. /mar.; $10(1): 113-122$.

26. KUMAR GA, et al. A population-based study of neonatal mortality and maternal care utilization in the Indian state of Bihar. BMC Pregnancy Childbirth. 2014; 14:357.

27. United Nations Children's Fund. Levels and trends in child mortality. Report 2015. Estimates Developed by the UN Inter-agency Group for Child Mortality Estimation [Internet]. Geneva: UNICEF; 2015 [cited 2016 Aug 09].

28. MARTINELLI KG, et al. Adequação do processo da assistência pré-natal segundo os critérios do Programa de Humanização do Pré-natal e Nascimento e Rede Cegonha. Rev. Bras. Ginecol. Obstet. 2014, 36(2):56-64.

29. ALVES DP, et al. Caracterização de gestantes em atendimento pré-natal. Revista da Universidade Vale do Rio Verde, Três Corações, 2015, 13(2):630- 638.

30. GAIOSO SEM et al. Gestantes atendidas em hospital de referência do SUS: quem são e quais os motivos? Journal of Management and Primary Health Care, 2014, 5 (1): 33-9.

31. GBD 2016 Mortality Collaborators. Global, regional, and national under-5 mortality, adult mortality, age-specific mortality, and life expectancy, 1970-2016: a systematic analysis for the Global Burden of Disease Study 2016. Lancet. 2017;390(10100):1084-150.

32. Galvão CM. Evidence Hierarchies. Acta Paul Enferm [Internet]. 2006[cited 2018 Feb 18];19(2):VI.

33. VOGEL JP, et al. Maternal complications and perinatal mortality: findings of the World Health Organization Multicountry Survey on Maternal and Newborn Health. BJOG: An International Journal of Obstetrics \& Gynaecology, 2019. Volume121, Issue s1.

34. LAOPAIBOON M, et al. Advanced maternal age and pregnancy outcomes: a multicountry assessment. An internation journal of Obstetric and Gynaecology, 2014; 121 (Suppl. 1):pp.49-56.

35. PASUPATHY D, et al. Advancedmaternal age and the risk of perinatal death due to intra-partum anoxia at term. $J$ Epidemiol Community Health2011;65:241-5.

36. VIELLAS EF, et al. Assistência pré-natal no Brasil. Cad Saúde Pública. 2014; 30 [Supl.1]: S85-S100

37. Brasil. Ministério da Saúde. Gabinete do Ministro. Portaria no 1459, de 24 de junho de 2011. Instituiu no âmbito do Sistema Único de Saúde - SUS - a Rede Cegonha. 2011.

38. SAMPAIO AFS, et al. Gestação de alto risco: perfil clínicoepidemiológico das gestantes atendidas no serviço de prénatal da maternidade pública de Rio Branco, Acre. Rev. Bras. Saúde Mater. Infant. vol.18 no.3 Recife jul./set. 2018, pp. 1-8. http://dx.doi.org/10.1590/1806-93042018000300007

39. MARTINS MCF, et al. (2019) Pregnancies with an outcome of fetal death present higher risk of delays in obstetric care: A case-control study. PLoS ONE 14(4): e0216037. 